\title{
THERMAL EMITTANCE MEASUREMENTS FROM NEGATIVE ELECTRON AFFINITY PHOTOCATHODES*
}

\author{
I.V. Bazarov, B.M. Dunham, F. Hannon ${ }^{\dagger}$, Y. Li, X. Liu, T. Miyajima ${ }^{\ddagger}$, D.G. Ouzounov, C.K. Sinclair \\ Cornell University, Ithaca, NY 14853, USA
}

\section{Abstract}

Recent computational optimizations have demonstrated that it should be possible to construct electron injectors based on photoemission cathodes in very high voltage DC electron guns in which the beam emittance is dominated by the thermal emittance from the cathode. Negative electron affinity (NEA) photocathodes are known to have a naturally low thermal emittance. However, the thermal emittance depends on the illuminating wavelength, the degree of negative affinity, and the band structure of the photocathode material. As part of the development of a high brightness, high average current photoemission electron gun for the injector of an ERL light source, we have measured the thermal emittance from negative affinity GaAs and GaAsP photocathodes. The measurements were made by obtaining the electron beam spot size downstream of a counter-wound solenoid lens as a function of the lens strength. Electron beam spot sizes were measured by two techniques: a $20 \mu \mathrm{m}$ wire scanner, and a $\mathrm{BeO}$ screen. Excellent agreement in measured emittance has been obtained between these two methods and direct transverse phase space mapping using a $20 \mu \mathrm{m}$ slit and the screen. Results are presented for both cathode types. The Cornell ERL injector performance is evaluated based on the results of these measurements.

\section{INTRODUCTION}

Cornell University is planning an ultra-bright $\mathrm{x}$-ray light source based on the Energy Recovery Linac (ERL) concept. The key component of the ERL is a high average current, low emittance electron injector. To this end, Cornell has an ongoing program to develop a high brightness injector system based on high voltage DC gun technology. The DC gun has been built and is now operational [1]. To achieve the required low emittance, the transverse emittance degradation due to space charge has to be controlled. Simulation studies have shown that it should be possible to achieve a very high degree of emittance compensation in the Cornell ERL injector so that the final beam emittance is dominated by thermal emittance from the cathode [2]. An ideal photocathode, in addition to high quantum efficiency and robustness, would have very low thermal emittance and a short photoemission response time, the latter to take advantage of laser pulse shaping to mitigate nonlinear

\footnotetext{
* Supported by the NSF grant PHY-0131508 and NSF/NIH-NIGMS award DMR-0225180

$\dagger$ visiting from TJNAF, Newport News, VA; Lancaster University, UK

$\ddagger$ visiting from Photon Factory, KEK, Tsukuba, Japan
}

space charge [3, 4]. NEA photocathodes have been shown to have a naturally low thermal emittance. However, the thermal emittance depends on the illuminating wavelength, the degree of negative affinity, and the band structure of the photocathode material. Furthermore, GaAs photocathodes have been measured to have a relatively long photoemission tail when excited with photons near the bandgap[5]. Since absorption in GaAs is a strong function of photon energy, the use of shorter radiation wavelengths for excitation may be preferable due to the significantly faster photoemission response time. The optimum wavelength will depend on the detailed behavior of thermal emittance and photoemission response versus laser wavelength. An effort is underway to characterize the response time from various photocathode materials at wavelengths of interest.

Here we present a careful study of the thermal emittance from two types of negative electron affinity photocathodes, GaAs and GaAsP, versus the laser wavelength. We also present the optimal performance of the Cornell ERL injector operating with these photocathodes illuminated with a frequency doubled $\mathrm{Yb}$-fiber laser system [6].

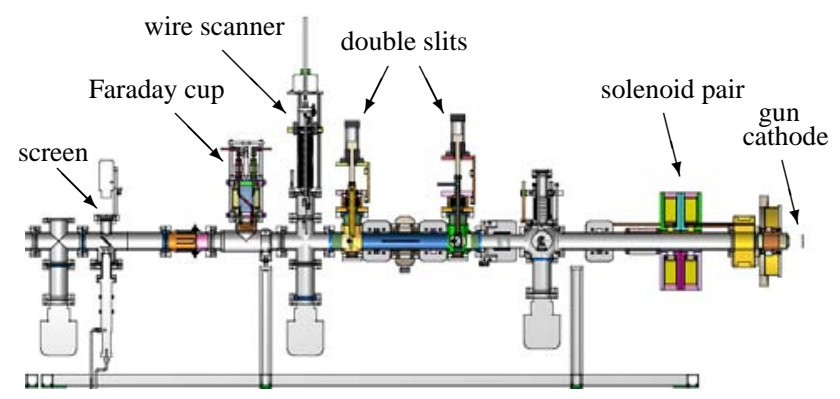

Figure 1: Beamline for thermal emittance studies. Beam direction is to the left.

\section{EXPERIMENTAL SETUP}

To commission the high-voltage DC gun and perform studies of the photocathodes and space charge dominated beam, a small beamline has been put together capable of transporting up to $100 \mathrm{~mA} \mathrm{DC}$ beam current. The beamline section directly after the gun is used for thermal emittance studies, and is shown in Fig. 1. The identical backto-back solenoids are located after the gun. The beamline is equipped with $20 \mu \mathrm{m}$ double slits for emittance measurement of space charge dominated beam. The wire scanner, strung with $20 \mu \mathrm{m}$ gold-plated tungsten wire, is positioned after the double slits, and is used to determine the beam 
profile. The current signal from the wire is connected to a trans-impedance amplifier and sampled together with the encoder reading at a $500 \mathrm{~Hz}$ rate. We have also used a $\mathrm{BeO}$ screen to visualize the electron beam and perform beam profile measurements. The view-port is equipped with a 12-bit CCD camera interfaced to a computer. The experiment is controlled via EPICS and MATLAB ${ }^{\circledR}$.

Four different lasers have been employed to cover the desired range of wavelengths: an argon-ion laser $(460 \mathrm{~nm})$, a solid-state green $5 \mathrm{~mW}(532 \mathrm{~nm})$, a helium-neon laser $(633 \mathrm{~nm})$, and a Ti:sapphire laser $(710-860 \mathrm{~nm})$. Similar to [7], a laser-illuminated aperture is imaged 1:1 on the photocathode with an achromatic 1-m focal length lens. Additionally, each time a thermal emittance measurement was taken, the laser beam was diverted after the lens and imaged at the same distance from the lens as the photocathode using a SPIRICON®12-bit CCD camera. A typical laser spot image is shown in Fig. 2a.

GaAs wafers with $\mathrm{Zn}$ doping between $2 \times 10^{18}$ and $2 \times{ }^{19} \mathrm{~cm}^{-3}$ have been activated in the preparation chamber of a load-lock system connected to the gun. A yo-yo preparation with cesium and nitrogen trifluoride gave typical quantum efficiencies of $10 \%$ at $532 \mathrm{~nm}$. GaAsP photocathodes have been activated in a similar fashion. GaAsP was epitaxially grown on GaAs substrates to a thickness of $2 \mu \mathrm{m}$. The phosphorus concentration was $45 \%$ with a $p$ doping level of $2-4 \times 10^{18} \mathrm{~cm}^{-3}$. A $2 \mu \mathrm{m}$ transition layer with graded phosphorus concentration separated the GaAs substrate and the GaAsP active layer.
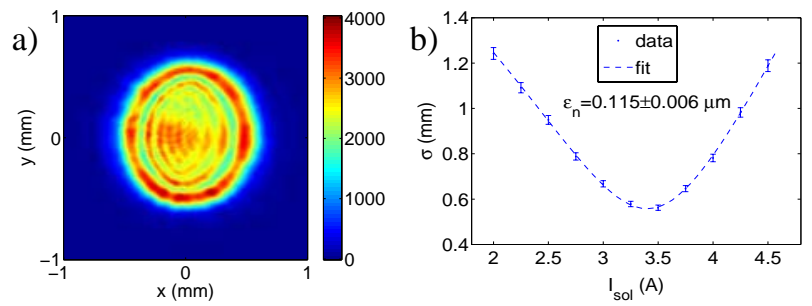

Figure 2: Sample data: a) measured laser profile; b) solenoid scan and fit $(860 \mathrm{~nm}, \mathrm{GaAs})$.

\section{MEASUREMENT METHOD}

A standard procedure for the case of decoupled motion in absence of space charge and beam dispersion was used to fit the transverse beam sizes measured as a function of the solenoid lens strength [8]. The transfer matrices were calculated for the actual magnetic field profile of the two back-to-back identical solenoids (see Fig. 3).

Fig. $2 \mathrm{~b}$ shows a typical solenoid scan and the fit. Most measurements were carried out at $250 \mathrm{kV}$ gun voltage. A cross-check for emittance was carried out at 200 and $250 \mathrm{kV}$ yielding the same normalized emittance within $1 \%$.

All beam profile data taken with the wire scanner or the screen was fitted with a super-Gaussian $y=$ $A e^{-\left(x-x_{0}\right)^{n} / s^{n}}+B$ with free parameters $A, B, n, s$, and $x_{0}$. The rms beam size is given by $s \sqrt{\Gamma\left(\frac{3}{n}\right) / \Gamma\left(\frac{1}{n}\right)}$. The 02 Synchrotron Light Sources and FELs

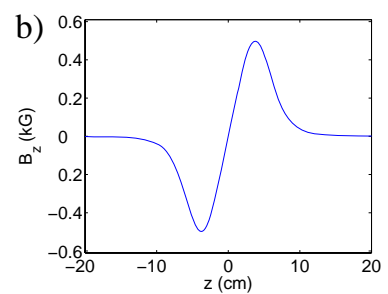

Figure 3: Solenoid field with 5 A excitation current: a) calculated and measured magnetic field of a single solenoid; b) magnetic field of two back-to-back identical solenoids.

largest error in determining the beam size is estimated to be $2 \%$. The measurement uncertainty was propagated in a $\chi^{2}$-fit to the emittance [8]. Typical errors on the emittance add up to $5 \%$ for the wire scanner and the screen, and $7 \%$ for the slit and screen technique.

The transverse thermal energy of the cathode $k T_{\perp}$, which relates to the rms normalized emittance $\epsilon_{n}$ according to

$$
\epsilon_{n}=\sigma_{0} \sqrt{\frac{k T_{\perp}}{m c^{2}}}
$$

$\sigma_{0}$ being the laser spot size and $m c^{2}$ the electron rest energy, was obtained from the slope $b$ of the $\chi^{2}$-fit of the data to $\epsilon_{n}=b \sigma_{0}$. The errors in both the laser spot $\sigma_{0}$ and the normalized emittance $\epsilon_{n}$ were taken into account. The typical uncertainty in determination of $\sigma_{0}$ was estimated to be $1 \% \mathrm{rms}$. The rms error to the slope $b$ was determined from the $\Delta \chi^{2}=1$ condition.

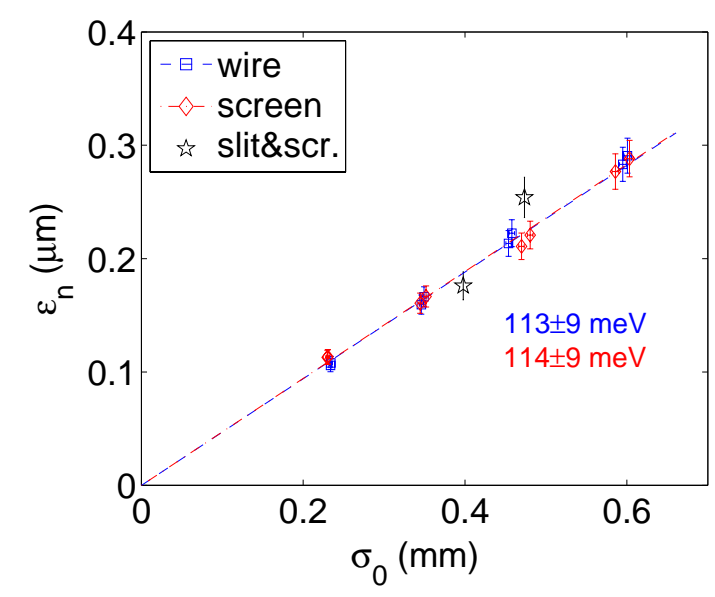

Figure 4: Comparison of various thermal emittance measurement techniques for GaAs at $532 \mathrm{~nm}$.

\section{RESULTS AND DISCUSSION}

Comparison of the thermal emittance as determined by the three techniques for GaAs at $532 \mathrm{~nm}$ is shown in Fig. 4, demonstrating a very good agreement.

Fig. 5 and 6 show the result of the thermal emittance measurements at various wavelengths for GaAs and GaAsP photocathodes respectively. The thermal energy

T02 Lepton Sources 


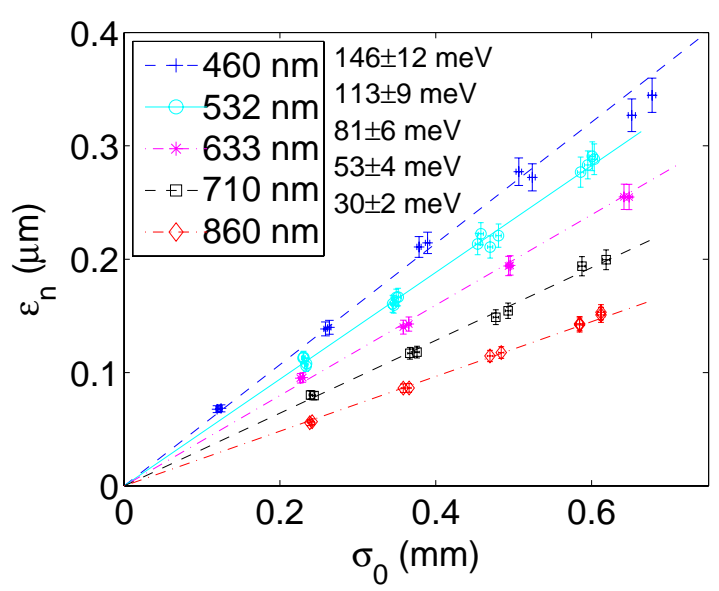

Figure 5: Measured thermal emittance for GaAs.

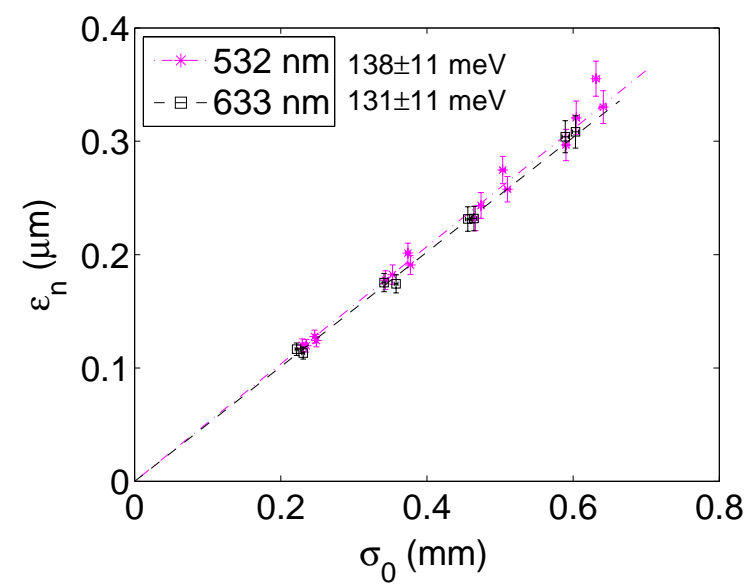

Figure 6: Measured thermal emittance for GaAsP.

at $532 \mathrm{~nm}$, near to the actual laser system wavelength of $520 \mathrm{~nm}$ [6], is found to be $113 \pm 9 \mathrm{meV}$ for GaAs, and $138 \pm 11 \mathrm{meV}$ for GaAsP. The bandgap of GaAsP, which undergoes a transition from direct to indirect gap for $45 \%$ phosphorus concentration, is closer matched to the laser wavelength than GaAs. Thus, the thermal energy of GaAsP was expected to be lower than that of pure GaAs at $532 \mathrm{~nm}$. Nevertheless, a larger $k T_{\perp}$ was obtained from GaAsP photocathodes. At the moment, the large thermal energy from GaAsP photocathodes is not understood, but may reflect a large fraction of the emitting electrons arising from the $\mathrm{X}$, rather than $\Gamma$ minimum.

To investigate the implications of the measured thermal emittance of the photocathode, a simulation study similar to [2] has been carried out for the now fully designed Cornell ERL injector. The normalized transverse emittance at the location of emittance diagnostic instrumentation at the end of the full injector $9.5 \mathrm{~m}$ from the cathode is shown in Fig. 7 as a function of the final bunch length $\sigma_{z}$ for a conservative value of $k T_{\perp}=150 \mathrm{meV}$. Three different gun voltages were investigated: 350,500 , and $750 \mathrm{kV}$. The average kinetic energy of the beam at the end of the beamline is $11 \mathrm{MeV}$. The initial laser distribution was assumed to be a uniform cylinder consistent with the pulse shaping techniques applied to the laser system. The average laser pulse 02 Synchrotron Light Sources and FELs

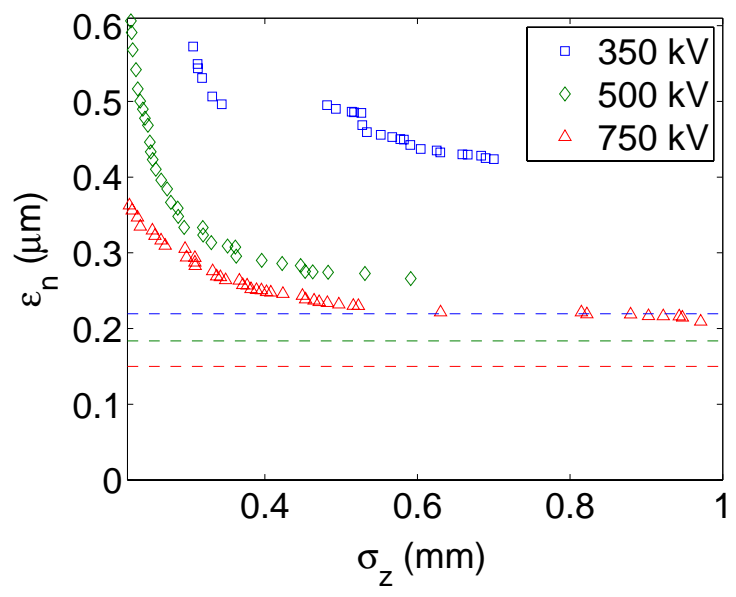

Figure 7: Performance of the DC gun injector with $77 \mathrm{pC}$ bunches for a photocathode with $k T_{\perp}=150 \mathrm{meV}$.

duration at the cathode is $13 \mathrm{ps}$ rms. This pulse duration and temporal shaping is consistent with photoemission response of GaAs at $520 \mathrm{~nm}$ [9].

The dashed lines in Fig. 7 show the theoretical limit to the emittance from the full injector at three different gun voltages for bunched electron beams applicable to our injector. The limit to the lowest normalized emittance $\epsilon_{n}$ assuming a flat-top transverse laser shape is given by

$$
\epsilon_{n}=\sqrt{\frac{Q \cdot k T_{\perp}}{4 \pi \varepsilon_{0} E_{\mathrm{cath}} m c^{2}}},
$$

here $Q$ is the bunch charge, $E_{\text {cath }}$ is the electric field at the cathode, $\varepsilon_{0}$ is the vacuum permittivity. It is seen from Fig. 7 that performance close to the theoretical limit can be achieved at both 500 and $750 \mathrm{kV}$ gun voltage.

\section{ACKNOWLEDGMENTS}

We acknowledge John Dobbins and John Barley for technical support with the control system, and Alexander Temknykh for magnetic measurements of the solenoids.

\section{REFERENCES}

[1] B.M. Dunham et al., "Performance of a very high voltage photoemission electron gun for a high brightness, high average current ERL injector", these proceedings

[2] I.V. Bazarov and C.K. Sinclair, PRST-AB, 8 (2005) 034202

[3] I.V. Bazarov et al., Proceedings of the 2005 Particle Accelerator Conference, IEEE 0-7803-8859-3 (2005) 2188

[4] S. Zhou et al., "Efficient temporal shaping of ultrashort pulses with birefringent crystals", submitted to J. Appl. Opt.

[5] P. Hartmann et al., J. Appl. Phys., 86 (1999) 2245

[6] D.G. Ouzounov et al., "The laser system for the ERL electron source at Cornell University", these proceedings

[7] B.M. Dunham et al., Proceedings of the 1995 Particle Accelerator Conference, IEEE 0-7803-3053-6 (1996) 2188

[8] M.G. Minty and F. Zimmermann,"Measurement and control of charged particle beams", Springer 2003

[9] Initial measurements of the response time from GaAs at Cornell gun development lab indicate a ps response in the green. 